\title{
Winds of M-type AGB stars driven by micron-sized grains
}

\author{
S. Höfner
}

\begin{abstract}
Department of Physics \& Astronomy, Division of Astronomy \& Space Physics, Uppsala University, Box 515, 75120 Uppsala, Sweden e-mail: Susanne.Hoefner@fysast.uu.se
\end{abstract}

Received 18 July 2008 / Accepted 11 September 2008

ABSTRACT

\begin{abstract}
Context. In view of the recent problem regarding the dynamical modelling of winds of M-type AGB stars (insufficient radiation pressure on silicate grains), some of the basic assumptions of these models need to be re-evaluated critically.

Aims. Accepting the conclusion that non-grey effects will force silicate grains to be virtually Fe-free, the viability of driving winds with micron-sized Fe-free silicates, instead of small particles, is examined.

Methods. Using both simple estimates and detailed dynamical atmosphere and wind models, it is demonstrated that radiation pressure on Fe-free silicate grains is sufficient to drive outflows if the restriction to the small particle limit is relaxed, and prevailing thermodynamic conditions allow grains to grow to sizes in the micrometer range.

Results. The predicted wind properties, such as mass loss rates and outflow velocities, are in good agreement with observations of M-type AGB stars. Due to a self-regulating feedback between dust condensation and wind acceleration, grain growth naturally comes to a halt at particle diameters of about $1 \mu \mathrm{m}$.

Conclusions. The most efficient grain sizes to drive winds are in a rather narrow interval around $1 \mu \mathrm{m}$. These values are set by the wavelength range corresponding to the flux maximum in typical AGB stars, and are very similar to interstellar grains.
\end{abstract}

Key words. stars: AGB and post-AGB - stars: mass-loss - stars: atmospheres - stars: winds, outflows - circumstellar matter dust, extinction

\section{Introduction}

Mass loss through slow stellar winds is a decisive feature of AGB stars which influences both their evolution and their observable properties. The basic scenario for this process is that of a pulsation-enhanced dust-driven wind: stellar pulsation triggers propagating atmospheric shock waves which intermittently lift gas above the stellar surface, creating dense, cool layers where solid particles may form. The grains are accelerated away from the star by radiation pressure, dragging the gas along. The composition of the dust is determined by the relative elemental abundances in the stellar atmosphere, with C-rich stars forming mostly amorphous carbon grains, and M-type objects producing silicate particles.

For the case of C-rich AGB stars, this scenario has successfully passed many tests, e.g., comparisons of detailed selfconsistent dynamical models with observations as diverse as low-resolution IR spectra (e.g., Gautschy-Loidl et al. 2004) and line profile variations of $\mathrm{CO}$ vibration-rotation lines (e.g., Nowotny et al. 2005).

However, similar models for M-type AGB stars (Woitke 2006; Höfner \& Andersen 2007) recently demonstrated two things: (i) silicate particles will be basically Fe-free due to nongrey effects on the grain temperature which were not apparent in earlier grey models (e.g., Jeong et al. 2003); and (ii) small Fe-free silicate particles result in insufficient radiative pressure to drive a wind since they are rather transparent at wavelengths around $1 \mu \mathrm{m}$, corresponding to the flux maximum in AGB stars.

In this paper, the cross-section relevant for radiative pressure on $\mathrm{Fe}$-free silicates is examined for a wide range of particle sizes, and the viability of micrometer-sized grains as wind drivers in
M-type AGB stars is demonstrated, both with simple estimates and with detailed radiation-hydrodynamical models.

\section{Silicates: $\mathrm{Mg} / \mathrm{Fe}$ and grain size}

To be considered for the short list of candidate AGB star wind drivers, any grain type must

- have a sufficiently high flux mean opacity per mass;

- form in sufficient quantities in the challenging environment of a pulsating atmosphere.

The first condition can be expressed as

$\kappa_{\mathrm{H}}>\kappa_{\text {crit }}=\frac{4 \pi c G M_{\star}}{L_{\star}}$

where $\kappa_{\mathrm{H}}$ denotes the flux mean opacity (cross-section per mass), and $M_{\star}$ and $L_{\star}$ are the stellar mass and luminosity, respectively, with $c$ and $G$ representing the speed of light and the gravitation constant.

The second condition is somewhat more complicated to specify as a quantitative criterion but it can be broken down into two separate estimates, i.e., how close to the stellar surface a certain grain type can form/survive, and how fast the grains (and, consequently, the amount of dust) will grow in given thermodynamical and chemical conditions. The condensation distance is mainly a question of grain temperature, while the growth rate and the maximum possible abundance of a dust type depend on the density and abundances of raw material for grain formation. Typical element abundances, together with observations of characteristic spectral features, lead to the expectation that silicates in the form of olivine, i.e. $\mathrm{Mg}_{2 x} \mathrm{Fe}_{2(1-x)} \mathrm{SiO}_{4}(0 \leq x \leq 1)$, and/or 
pyroxene, i.e. $\mathrm{Mg}_{x} \mathrm{Fe}_{(1-x)} \mathrm{SiO}_{3}$, are the dominant dust species in M-type AGB stars.

A grain's temperature will be mostly determined by its interaction with the radiation field. We can make a simple estimate for the condensation radius $R_{\mathrm{c}}$ by defining it as the distance where the radiative equilibrium temperature of a grain is equal to the condensation temperature $T_{\mathrm{c}}$ of the material. Assuming a Planckian radiation field, geometrically diluted with distance from the star, and a power law for the grain absorption coefficient, $\kappa_{\lambda} \propto \lambda^{-p}$, in the relevant wavelength range (around the flux maximum of the star) the condensation radius can be expressed as

$$
\frac{R_{\mathrm{c}}}{R_{*}}=\frac{1}{2}\left(\frac{T_{\mathrm{c}}}{T_{*}}\right)^{-\frac{4+p}{2}}
$$

(cf. Höfner 2007, and references therein for details). This implies that silicates forming in atmospheres of AGB stars $\left(T_{\mathrm{c}} \approx\right.$ $1000 \mathrm{~K}, T_{\star} \approx 3000 \mathrm{~K}$ ) have to be virtually iron-free, as the inclusion of Fe leads to $p \approx 2$ and, consequently, to $R_{\mathrm{c}} / R_{\star}>10$, whereas iron-free silicates $(p \approx-1)$ can form at typically $R_{\mathrm{c}} / R_{\star} \approx 2-3$. These numbers are in good agreement with detailed models (Woitke 2006; Höfner \& Andersen 2007).

Having concluded that the silicates will be Fe-free, we are faced with the fact that such grains are nearly transparent at wavelengths around $1 \mu \mathrm{m}$, meaning that their absorption cross section $C_{\text {abs }}$ is very low. In the case of particles being small compared to the relevant wavelengths, the grain cross section determining the radiative pressure,

$C_{\mathrm{rp}}=C_{\mathrm{ext}}-g_{\mathrm{sca}} C_{\mathrm{sca}}=C_{\mathrm{abs}}+\left(1-g_{\mathrm{sca}}\right) C_{\mathrm{sca}}$

(where $g_{\text {sca }}$ is the mean of the cosine of the angle of scattering; cf., e.g., Krügel 2003), is dominated by the extinction cross section $C_{\text {ext }}$, which in turn is equivalent to the absorption cross-section $C_{\text {abs }}=C_{\text {ext }}-C_{\text {sca }}$, as scattering is negligible $\left(C_{\mathrm{sca}} / C_{\mathrm{ext}} \ll 1\right)$ in this limit. This leads to the previously discussed problem of insufficient opacity for driving winds. As grains become larger and approach sizes comparable to the wavelength of interest, however, scattering plays an increasingly important role, and starts to dominate $C_{\mathrm{rp}}$.

While cross-sections as such are useful for comparing individual grains of different sizes and materials, we need the total opacity (cross section per mass), $\kappa_{\mathrm{rp}}\left(\lambda, a_{\mathrm{gr}}\right)$, of all grains of a certain radius $a_{\text {gr }}$ in a matter element (with a total mass density $\rho$ ) at wavelength $\lambda$ to estimate their contribution to the radiative pressure. Introducing the efficiency $Q_{\mathrm{rp}}=C_{\mathrm{rp}} / \pi a_{\mathrm{gr}}^{2}$ and denoting the number density of grains of radius $a_{\mathrm{gr}}$ by $n_{\mathrm{gr}}$, we can write this opacity as

$\kappa_{\mathrm{rp}}\left(\lambda, a_{\mathrm{gr}}\right)=\frac{\pi}{\rho} \frac{Q_{\mathrm{rp}}\left(\lambda, a_{\mathrm{gr}}\right)}{a_{\mathrm{gr}}} a_{\mathrm{gr}}^{3} n_{\mathrm{gr}}$

where $a_{\mathrm{gr}}^{3} n_{\mathrm{gr}}$ represents the fraction of a given volume which is occupied by the grains, apart from a factor $4 \pi / 3$. This quantity can be rewritten in terms of the space occupied by a monomer (basic building block) in the condensed material times the number of monomers found in a certain volume,

$a_{\mathrm{gr}}^{3} n_{\mathrm{gr}}=\frac{3}{4 \pi} V_{\mathrm{mon}} n_{\mathrm{mon}}=\frac{3}{4 \pi} \frac{A_{\mathrm{mon}} m_{\mathrm{p}}}{\rho_{\text {grain }}} f_{\mathrm{c}} \varepsilon_{\mathrm{c}} n_{\mathrm{H}}$

where we have expressed the monomer volume $V_{\text {mon }}$ in terms of the atomic weight of the monomer $A_{\text {mon }}$ and the density of

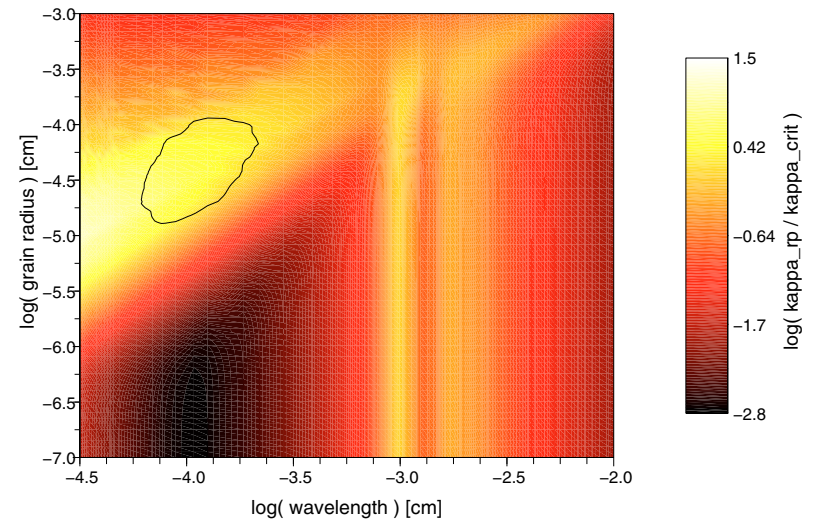

Fig. 1. Opacity $\kappa_{\text {rp }}$ of forsterite grains in units of the critical opacity $\kappa_{\text {crit }}$ $\left(M_{\star}=1 M_{\odot}, L_{\star}=7000 L_{\odot}\right)$, assuming that 30 percent of Si is condensed into grains; The black contour shows the region where the fluxweighted opacity exceeds the critical opacity, assuming a Planckian flux distribution with $T_{\star}=2700 \mathrm{~K}$.

the grain material $\rho_{\text {grain }}$, and the number of monomers in a volume $n_{\text {mon }}$ by the abundance of the key element of the condensate $\varepsilon_{\mathrm{c}}$, the degree of condensation of this key element $f_{\mathrm{c}}$, and the total number density of $\mathrm{H}$ atoms $n_{\mathrm{H}}\left(m_{\mathrm{p}}=\right.$ proton mass). Using $n_{\mathrm{H}}=\rho /\left(1+4 \varepsilon_{\mathrm{He}}\right) m_{\mathrm{p}}$, we obtain

$\kappa_{\mathrm{rp}}\left(\lambda, a_{\mathrm{gr}}\right)=\frac{3}{4} \frac{A_{\mathrm{mon}}}{\rho_{\text {grain }}} \frac{Q_{\mathrm{rp}}\left(\lambda, a_{\mathrm{gr}}\right)}{a_{\mathrm{gr}}} \frac{\varepsilon_{\mathrm{c}}}{1+4 \varepsilon_{\mathrm{He}}} f_{\mathrm{c}}$.

Figure 1 shows the opacity $\kappa_{\mathrm{rp}}$ of $\mathrm{Fe}$-free olivine grains (forsterite, $\mathrm{Mg}_{2} \mathrm{SiO}_{4}$ ) as a function of wavelength and grain radius $a_{\text {gr }}$ relative to the critical opacity $\kappa_{\text {crit }}$ (Eq. (1)), assuming that 30 percent of the $\mathrm{Si}$ are condensed into dust $\left(\varepsilon_{\mathrm{Si}}=\right.$ $\left.3.55 \times 10^{-5}, \varepsilon_{\mathrm{He}}=0.1, A_{\text {mon }}=140, \rho_{\text {grain }}=3.27 \mathrm{~g} / \mathrm{cm}^{3}\right)$. The quantity $Q_{\mathrm{rp}}$ has been calculated using Mie theory for spherical particles (program BHMIE from Bohren \& Huffman 1983, modified by Draine, www.astro.princeton.edu/ draine/ scattering.html) and refractive index data by Jäger et al. (2003). The critical opacity is taken for a stellar mass of $1 M_{\odot}$ and a luminosity of $7000 L_{\odot}$. For wavelengths around $1 \mu \mathrm{m}$, starting from very low values for small grains, the opacity reaches a pronounced maximum for particles of $a_{\mathrm{gr}} \approx 0.3 \mu \mathrm{m}$, and then decreases again. Looking at the actual values of the opacity, there are several regions where it clearly exceeds the critical opacity: a diagonal band where $a_{\mathrm{gr}}$ is about equal to the wavelength, and two vertical zones, corresponding to two maxima of $Q_{\mathrm{rp}}$ near $10 \mu \mathrm{m}$ which produce the characteristic silicate feature in spectra of M-type AGB stars.

In order to decide which grains will contribute to driving a wind, however, the opacity needs to be weighted with the relative flux as a function of wavelength. For simplicity, we assume that the stellar flux can be approximated by a Planck function, and we define the monochromatic flux-weighted opacity as

$\tilde{\kappa}_{\mathrm{rp}}\left(\lambda, a_{\mathrm{gr}}, T_{\star}\right)=\kappa_{\mathrm{rp}}\left(\lambda, a_{\mathrm{gr}}\right) \frac{\lambda B_{\lambda}\left(T_{\star}\right)}{T_{\star}^{4} \sigma / \pi}$.

The black contour in Fig. 1 shows the region where this opacity exceeds the critical opacity for $T_{\star}=2700 \mathrm{~K}$. This last criterion limits the effective sizes for grains to a rather narrow interval around $a_{\mathrm{gr}} \approx 0.3 \mu \mathrm{m}$. Only grains in this size range can drive the wind, and a considerable fraction of the available material has to be contained in these grains to push the total opacity above the critical value. 
So far, we have deduced that silicate grains in M-type AGB stars have to be virtually Fe-free to bring the condensation distance down well below $10 R_{\star}$, and they have to have particle radii in the range of about $0.1-1.0 \mu \mathrm{m}$ to achieve a large radiative cross-section. The remaining question is, whether sufficient amounts of material are able to condense into grains in the variable conditions of the stellar atmosphere. While it is possible to make simple estimates for grain growth time scales based on densities, abundances, and grain properties (cf. Gustafsson \& Höfner 2004), this question will be answered with detailed wind models presented in the next section.

\section{Detailed dynamical models}

In order to test the hypothesis of micron-sized Fe-free silicate grains as wind drivers in a reasonably realistic setting, the dynamical atmosphere and wind models of Höfner et al. (2003) are adapted by including routines which compute frequencydependent dust opacities for arbitrary grain sizes (cf. Sect. 2). In contrast to earlier versions, the models contain a time-dependent description of grain growth for silicate grains and they are based on radiative transfer in 319 wavelength points (instead of 64), to ensure a better coverage of the complex radiation field, taking both molecular and grain opacities into account. In the present context, we consider the formation of pure forsterite particles according to the net reaction

$2 \mathrm{Mg}+\mathrm{SiO}+3 \mathrm{H}_{2} \mathrm{O} \longrightarrow \mathrm{Mg}_{2} \mathrm{SiO}_{4}+3 \mathrm{H}_{2}$.

The grain growth rate is calculated following Gail \& Sedlmayr (1999), assuming that the step determining the total rate is the addition of a $\mathrm{SiO}$ molecule to the grain surface. The vapor pressure of $\mathrm{SiO}$ over olivine is based on data for free energies from Sharp \& Huebner (1990).

While treating the growth of grains with a detailed timedependent description, the nucleation of new grains from the gas phase is not considered, assuming the presence of seed nuclei (a given number per $\mathrm{H}$ atom, $n_{\mathrm{gr}} / n_{\mathrm{H}}$ ) at the point where grain growth becomes possible. At present, this is necessary due to the lack of nucleation rates for such grains. The value of $n_{\mathrm{gr}} / n_{\mathrm{H}}$ is varied within (but not fully covering) a range which, in principle, allows particles to grow to sizes up to and beyond the micrometer range. At the upper end of the viable $n_{\mathrm{gr}} / n_{\mathrm{H}}$ range (many particles), grain growth will be limited by the availability of material, resulting in small grains and, consequently, a too low radiative cross-section to drive a wind. At the lower end (few particles), grains may grow quickly beyond the efficient size range to drive a wind (see contour in Fig. 1), leading to a rather low total opacity, even for high degrees of condensation. Since the relevant interval of grain radii is about one order of magnitude, the corresponding range in $n_{\mathrm{gr}} / n_{\mathrm{H}}$ should span about 3 orders of magnitude for a given $f_{\mathrm{c}}$ (cf. Eq. (5)).

It should be noted that the actual value of $a_{\mathrm{gr}}$ is a result of the models, not a parameter. For a given $n_{\mathrm{gr}} / n_{\mathrm{H}}$, the final grain radius is determined by the growth rates under the prevailing dynamical and thermodynamical conditions in the atmosphere and wind.

The parameters and resulting wind properties of the detailed models are listed in Table $1\left(\Delta u_{\mathrm{p}}=\right.$ velocity amplitude of piston). The letters A and B denote different combinations of stellar parameters, i.e., mass $M_{\star}$, luminosity $L_{\star}$, effective temperature $T_{\star}$ (initial model) and pulsation period $P$ (solar abundances, Grevesse et al. 1996)

\begin{tabular}{ccccc}
\hline \hline Model & $M_{\star}\left[M_{\odot}\right]$ & $L_{\star}\left[L_{\odot}\right]$ & $T_{\star}[\mathrm{K}]$ & $P[\mathrm{~d}]$ \\
\hline A & 1.0 & 5000 & 2800 & 310 \\
B & 1.0 & 7000 & 2700 & 390 \\
\hline
\end{tabular}
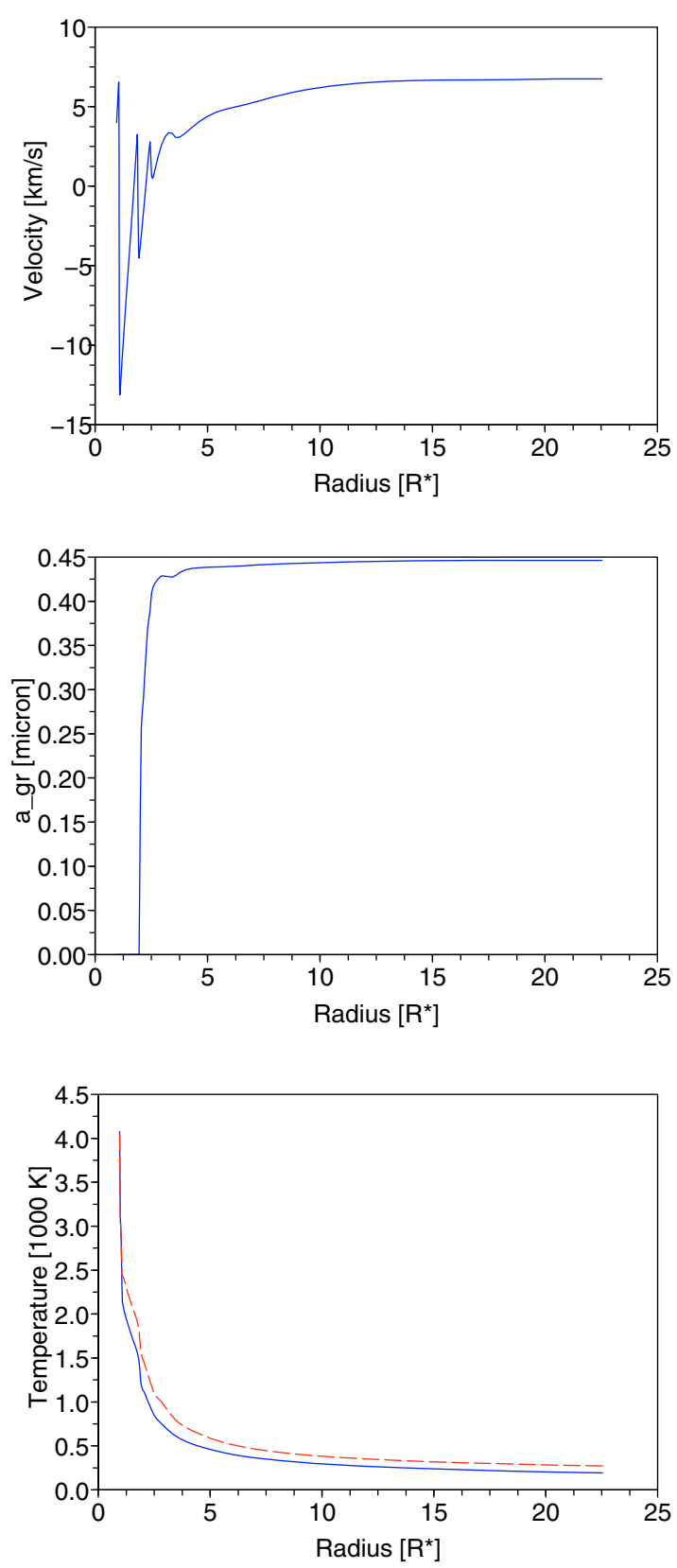

Fig. 2. Snapshot of the spatial structure of model B with $n_{\mathrm{gr}} / n_{\mathrm{H}}=1 \times$ $10^{-15}$ : top: velocity, middle: grain radius, bottom: grain temperature (solid) and gas temperature (dashed).

Table 1. Model parameters and resulting wind properties.

\begin{tabular}{ccccccc}
\hline \hline$*$ & $n_{\mathrm{gr}} / n_{\mathrm{H}}$ & $\begin{array}{c}\Delta u_{\mathrm{p}} \\
{\left[\mathrm{km} \mathrm{s}^{-1}\right]}\end{array}$ & $\begin{array}{c}\dot{M} \\
{\left[M_{\odot} / \mathrm{yr}\right]}\end{array}$ & $\begin{array}{c}\langle u\rangle \\
{\left[\mathrm{km} \mathrm{s}^{-1}\right]}\end{array}$ & $\left\langle f_{\mathrm{Si}}\right\rangle$ & $\begin{array}{c}\left\langle a_{\mathrm{gr}}\right\rangle \\
{[\mu \mathrm{m}]}\end{array}$ \\
\hline $\mathrm{A}$ & $1 \times 10^{-15}$ & 4.0 & $4 \times 10^{-7}$ & 5 & 0.18 & 0.48 \\
$\mathrm{~A}$ & $3 \times 10^{-15}$ & 4.0 & $8 \times 10^{-7}$ & 10 & 0.22 & 0.36 \\
$\mathrm{~B}$ & $3 \times 10^{-16}$ & 4.0 & $5 \times 10^{-7}$ & 4 & 0.14 & 0.66 \\
$\mathrm{~B}$ & $1 \times 10^{-15}$ & 4.0 & $8 \times 10^{-7}$ & 7 & 0.15 & 0.45 \\
$\mathrm{~B}$ & $3 \times 10^{-15}$ & 4.0 & $1 \times 10^{-6}$ & 11 & 0.20 & 0.34 \\
\hline
\end{tabular}

The resulting mass loss rates $\dot{M}$ and wind velocities $\langle u\rangle$ are in good agreement with observed values for M-type AGB stars (cf. Olofsson 2004, and references therein), and the predicted grain sizes $\left\langle a_{\mathrm{gr}}\right\rangle$ are in accordance with the simple estimates in 
Sect. 2. The fact that the mean degrees of condensation of $\operatorname{Si}\left\langle f_{\mathrm{Si}}\right\rangle$ are well below 0.5 (limit for forsterite due to $\mathrm{Mg}$ abundance) is a consequence of grain growth practically coming to a halt when sizes efficient for driving the wind are reached, due to the acceleration of the wind and the resulting fast decrease in gas densities. This is also illustrated by Fig. 2, showing a snapshot of the radial structure of a selected model.

\section{Conclusions}

Both simple estimates and results of detailed radiationhydrodynamical models presented in this article demonstrate that winds of M-type AGB stars can be driven by radiation pressure on micron-sized Fe-free silicate grains. Particles in this size range have a considerable radiative scattering cross-section which compensates for their low absorption cross-section, leading to efficient radiative acceleration. Resulting combinations of mass loss rates and wind velocities are in good agreement with observations, and a less than 30 percent fraction of Si condensed into forsterite is sufficient to drive winds for typical stellar parameters.

The results presented here are for solar abundances and olivine grains, but similar arguments should hold for pyroxene particles. Different chemical compositions of both the gas phase and the condensates will be discussed in a forthcoming paper. As C/O approaches 1 (S-type AGB stars), silicate formation will eventually be limited by the lack of oxygen not bound in $\mathrm{CO}$, rather than by the abundances of $\mathrm{Si}$ and $\mathrm{Mg}$. The fact that $\mathrm{S}$ stars show similar wind properties as M- and C-type AGB stars (Ramstedt et al. 2006) may indicate that some oxygen is made available for grain formation by partial breaking up of $\mathrm{CO}$ due to shocks. The scenario suggested by Höfner \& Andersen (2007) could be modified such that silicate grains contribute to or take over the wind-driving for stars with somewhat more oxygen than carbon.
The probably most attractive conclusion based on the current results is that silicate grains from AGB stars found in the interstellar medium should be expected to have diameters of the order of $1 \mu \mathrm{m}$ - provided they are driving the stellar outflow due to a self-regulating feedback between grain growth and wind acceleration. Particles produced in this way will fall into a rather narrow size range which coincides with observations of grains in the interstellar medium.

Acknowledgements. This work has been supported by the Swedish Research Council (Vetenskapsrådet).

\section{References}

Andersen, A. C., Höfner, S., \& Gautschy-Loidl, R. 2003, A\&A, 400, 981 Bohren, C. F., \& Huffman, D. 1983, Absorption and scattering of light by small particles (New York: John Wiley)

Gail, H.-P., \& Sedlmayr, E. 1999, A\&A, 347, 594

Gautschy-Loidl, R., Höfner, S., Jørgensen, U. G., \& Hron, J. 2004, A\&A, 422, 289

Gustafsson, B., \& Höfner, S. 2004, in Asymptotic Giant Branch Stars, ed. H. J. Habing, \& H. Olofsson (Springer)

Grevesse, N., Noels, A., \& Sauval, J. 1996, ASP Conf. Ser., 99, 117

Höfner, S. 2007, ASP Conf. Ser., 378, 145

Höfner, S., \& Andersen, A. C. 2007, A\&A, 465, L39

Höfner, S., Gautschy-Loidl, R., Aringer, B., \& Jørgensen, U. G. 2003, A\&A, 399,589

Jäger, C., Dorschner, J., Mutschke, H., Posch, Th., \& Henning, Th. 2003, A\&A, 408, 193

Jeong, K. S., Winters, J. M., Le Bertre, T., \& Sedlmayr, E. 2003, A\&A, 407, 191 Krügel, E. 2003, The Physics of Interstellar Dust, IoP

Nowotny, W., Aringer, B., Höfner, S., Gautschy-Loidl, R., \& Windsteig, W. 2005, A\&A, 437, 273

Olofsson, H. 2004, in Asymptotic Giant Branch Stars, ed. H. Habing, \& H. Olofsson (New York: Springer)

Ramstedt, S., Schöier, F. L., Olofsson, H., \& Lundgren, A. A. 2006, A\&A, 454, L103

Sharp, C. M., \& Huebner, W. F. 1990, ApJS, 72, 417

Woitke, P. 2006, A\&A, 460, L9 